\title{
Safety and efficiency of emergency department interrogation of cardiac devices
}

\author{
James F. Neuenschwander ${ }^{1}$, W. Frank Peacock ${ }^{2}$, Madgy Migeed $^{3}$, \\ Sara A. Hunter ${ }^{4}$, John C. Daughtery ${ }^{5}$, lan C. McCleese ${ }^{6}$, \\ Brian C. Hiestand ${ }^{7}$ \\ 'Department of Emergency Medicine, Genesis Health Care Systems, Zanesville, OH, USA \\ ${ }^{2}$ Department of Emergency Medicine, Baylor College of Medicine, Houston, TX, USA \\ ${ }^{3}$ Department Cardiology, Genesis Health Care Systems, Zanesville, OH, USA \\ ${ }^{4}$ Department of Radiology, The Cleveland Clinic Foundation, Cleveland, OH, USA \\ ${ }^{5}$ Department of Cell Biology and Physiology, Ohio State University Wexner Medical Center, Columbus, $\mathrm{OH}$, \\ USA \\ ${ }^{6}$ Ohio State University, Columbus, OH, USA \\ ${ }^{7}$ Department of Emergency Medicine, Wake Forest School of Medicine, Winston-Salem, NC, USA
}

\begin{abstract}
Objective Patients with implanted cardiac devices may wait extended periods for interrogation in emergency departments (EDs). Our purpose was to determine if device interrogation could be done safely and faster by ED staff.
\end{abstract}

Methods Prospective randomized, standard therapy controlled, trial of ED staff device interrogation vs. standard process (SP), with 30-day follow-up. Eligibility criteria: ED presentation with a self-report of a potential device related complaint, with signed informed consent. SP interrogation was by company representative or hospital employee.

Results Of 60 patients, 42 (70\%) were male, all were white, with a median (interquartile range) age of 71 (64 to 82) years. No patient was lost to follow up. Of all patients, 32 (53\%) were enrolled during business hours. The overall median (interquartile range) ED vs. SP time to interrogation was 98.5 ( 40 to 260 ) vs. 166.5 ( 64 to 412 ) minutes ( $P=0.013$ ). While ED and SP interrogation times were similar during business hours, 102 (59 to 138) vs. 105 (64 to 172) minutes $(P=0.62)$, ED interrogation times were shorter vs. SP during non-business hours; 97 (60 to 126) vs. 225 (144 to 412 ) minutes, $P=0.002$, respectively. There was no difference in $E D$ length of stay between the ED and SP interrogation, 249 (153 to 390) vs. 246 (143 to 333) minutes $(P=0.71)$, regardless of time of presentation. No patient in any cohort suffered an unplanned medical contact or post-discharge adverse device related event.

Conclusion ED staff cardiac device interrogations are faster, and with similar 30-day outcomes, as compared to SP.

Keywords Emergency medicine; Pacemaker; Safety; Defibrillators, implantable; Interrogation
elSSN: 2383-4625

Received: 19 December 2015

Revised: 6 January 2016

Accepted: 8 March 2016

Correspondence to:

James F. Neuenschwander

Genesis Healthcare Systems, 2951

Maple Avenue, Zanesville, $\mathrm{OH} 43701$, USA

E-mail: Jim.neuen@gmail.com

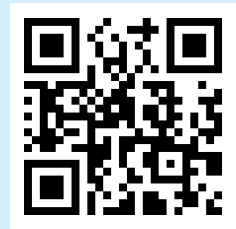

How to cite this article:

Neuenschwander JF, Peacock WF, Migeed M, Hunter SA, Daughtery JC, McCleese IC, Hiestand BC. Safety and efficiency of emergency department interrogation of cardiac devices. Clin Exp Emerg Med 2016;3(4):239-244.

This is an Open Access article distributed under the terms of the Creative Commons Attribution Non-Commercial License (http:// creativecommons.org/licenses/by-nc/3.0/). 


Capsule
$\begin{aligned} & \text { What is already known } \\ & \text { Patients often wait for prolonged periods of time to have their pacemakers and implantable cardioverter-defibrillators } \\ & \text { checked by industry representatives or hospital staff trained in interrogations. }\end{aligned}$
$\begin{aligned} & \text { What is new in the current study } \\ & \text { Emergency department staff can check these devices more quickly and just as safely and efficiently. }\end{aligned}$

\section{INTRODUCTION}

The rate of implantation of cardiac devices has increased markedly over the past twenty years. ${ }^{1}$ In the United States, approximately 370,000 implantable cardiac devices (pacemakers and implantable cardioverter-defibrillators [ICDs]) are implanted each year. ${ }^{2}$ As patients are living longer because of these devices, the number of patients with related complaints presenting to emergency departments (EDs) is likely to increase. ${ }^{3}$

Device interrogation is frequently required to provide diagnostic management; unfortunately, material and personnel resources may not be immediately available in the ED to interrogate the device. This delay can contribute to prolonged ED stays and crowding, which has been associated with adverse outcomes in a number of populations. ${ }^{4-7}$

Frequently, checking a device requires calling industry representatives or specially trained healthcare providers to bring device readers and perform the interrogation in the ED. As the various devices are not cross-compatible with readers from other manufacturers, there is also the potential for further delay if the wrong industry representative is called. This can occur if the patient does not know what type of device they have received, or if the medical record is not available to the ED to which the patient presented. Although registries are maintained by the 3 major manufacturers of the United States, querying all 3 is time consuming, and may be fruitless if the patient's device was manufactured by a smaller company. All of this complexity leads to decreased throughput for the patient and prolonged ED length of stay (LOS). In some facilities, this expected delay is managed by admitting the patient to an inpatient bed, which results in an increase in costs and represents an inefficient use of resources. Although registries are maintained of patients with devices, no information on the number of visits to EDs is formally kept so baseline frequencies of ED interrogations are not available at this time. Concern exists among cardiology and other members of hospital staff that EDs should not do interrogations of cardiac devices as the risk for inadvertent reprogramming or rendering the device non-functional is possible.
We have previously demonstrated that device interrogation can be performed by ED-based personnel. ${ }^{8}$ In this study, our hypothesis was that patients requiring interrogation would receive it more quickly and just as safely as when performed by ED personnel when compared to the standard process (SP) provided by specialized trained healthcare providers or manufacturer representatives. Safety is defined as relaying all needed information and not accidently reprogramming the device and placing the patient at risk due to programming inappropriate parameters or rendering it ineffective.

\section{METHODS}

\section{Study design}

Using a convenience sample of patients presenting to the ED with potentially cardiac device related complaints over every time period of the day, we performed a prospective, randomized SP-controlled study comparing device interrogation by ED personnel in the ED to that by cardiology nurses or device manufacturer personnel. SP was determined by the emergency physician that the device interrogation was necessary, and a consult to cardiology nursing staff trained in device interrogation or the device manufacturer representatives was requested. When interrogation was performed by cardiology nurses or device manufacturer, it could have occurred in the ED or after admission, dependent upon what time the interrogation personnel arrived at the patient's bedside.

\section{Study setting and population}

We conducted our study at a two ED community hospital system with a combined census of 70,000 visits a year. As programming devices are manufacturer specific, we limited our cohort to those patients presenting with a complaint related to a pacemaker or ICD manufactured by a single manufacturer (Boston Scientific, Marlborough, MA, USA). Patients were considered eligible for participation if they provided written informed consent, were believed to have a Boston Scientific device, and presented with a complaint consistent with a potential device malfunction. $\mathrm{Pa}$ - 
tients were excluded if, at the determination of the attending emergency physician, device interrogation was not required to make a diagnosis or treatment decision. All patients provided informed consent, and the study was approved by the institutional review board.

\section{Study protocol}

ED staff interrogation was performed by 13 local ED nurses who were trained on data acquisition using a BSC ZOOM programmer model 3120 (Boston Scientific) which was the standard model being utilized at that time. The training of the registered nurses took approximately 1 hour and was done by either the device manufacturer representative or the educational specialist at the facility. The training was free of charge. The training for ED staff to gather the data was considerably shorter than the months of training required by the specialized trained healthcare providers and company representatives. Our goal was to obtain information only, therefore the lengthy training could be truncated because no interpretation or reprogramming skills were needed. Because our training was simple and staff were motivated to participate, the time someone was available to perform the interrogation was nearly the entire length of the study both day and night. After acquisition, data was transmitted to the company representative on call, who shared it with the cardiologist on call. Alternatively, the ED staff could directly contact the cardiologist on call and forward the data output and clinical information. All data resulting from the device interrogation were available to ED and cardiology staff for clinical decision making. Post-interrogation medical care was determined by the clinical team and not dictated by protocol. SP device interrogation was per the local representative's routine and training.

\section{Data definitions and outcomes}

Normal business hours were defined as patient presentation to the ED between 6 a.m. and 5 p.m., Monday through Friday excluding US federal holidays as this is when company representatives and staff trained to do the interrogations are typically in the hospital and immediately available to help the ED. Data on time to interrogation, time to disposition decision (defined as an order for admission, discharge, or transfer to another facility), and ED LOS were recorded. Adverse events were defined as inadvertent reprogramming of a device, or any repeated hospitalization, or ED admission, or unplanned clinic visit caused by ED interrogation.

Inadvertent programming was defined as the accidental discontinuation of device functioning or changing device parameters to render it ineffective in serving the patient. Research staff contacted the patient by phone at 30 days post index visit, per- formed a review of the electronic medical record, and contacted the patient's physician to determine the incidence of adverse events subsequent to device interrogation. An electrophysiologist reviewed all the medical records for adverse events.

\section{Data analysis}

Medians are presented with interquartile ranges (IORs). Time data was found to be non-parametric using Shapiro-Wilk testing, therefore Wilcoxon Rank Sum analysis was used to compare median times between groups. Fisher's exact test was used for comparing proportions and categorical variables. We used Stata ver. 11.2 (Stata Corp., College Station, TX, USA) to complete the analyses. Alpha was defined as $\mathrm{P}<0.05$, and was not adjusted for multiple comparisons. Formal sample size analysis was not performed for this pilot analysis. Multiple patient visits were allowed and they could be randomized to either group each time.

\section{RESULTS}

Sixty patients were enrolled between July 1, 2011 and August 13, 2013 through all hours of the day and week, of which 32 were randomized to ED interrogation. In the study sample, 42 (70\%) were male, and all were white; the median age was 71 (IQR, 64 to 82; range, 36 to 95 years). Admission or transfer to a tertiary center was required in 25/32 (78\%) patients randomized to ED interrogation, vs. 19/28 (68\%) in the SP arm $(P=0.40)$. ED interrogation patients presented during normal business hours in $17 / 32(53 \%)$ cases, vs. $15 / 28(54 \%)$ of those randomized to SP $(P=1.0)$. Two patients in the SP group were enrolled more than once, twice in one case and three times in the other. Overall, the most common ED diagnoses for all patients at the index visit were ventricular tachycardia/fibrillation (15\%), ICD discharge (15\%), chest pain (8\%), atrial fibrillation (7\%), pacemaker malfunction (7\%), and congestive heart failure (5\%) (Fig. 1). No patients were lost to follow up.

The median time to ICD interrogation was 68 minutes shorter in the ED than SP group, 98.5 (40 to 260) vs. 166.5 (64 to 412) minutes $(P=0.013)$ (Fig. 2). During normal business hours, there was no difference between cohorts; 102 (59 to 138) vs. 105 (64 to 172) minutes $(P=0.62)$, for the ED vs. SP interrogation cohorts (Fig. 3). However, during non-business hours, median time to interrogation was substantially shorter in the ED group, 97 (60 to 126) vs. 225 (144 to 412) minutes for the SP group ( $P=0.002$ ) (Fig. 4).

There was no difference in the amount of time to disposition decision between the ED interrogation cohort vs. SP, 162 (72 to 258 ) vs. 156 (99 to 234 ) minutes ( $P=0.78$ ). Likewise, there was no difference in the total amount of time spent in the ED between the ED interrogation cohort vs. SP, 249 (153 to 390) vs. 246 (143 


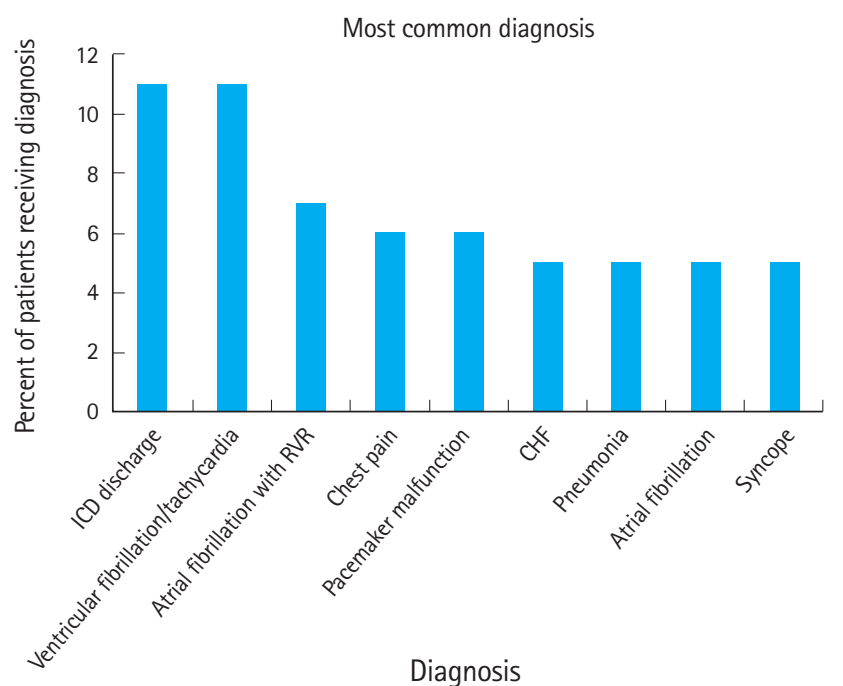

Fig. 1. Common diagnoses for the study cohort. ICD, implantable cardioverter defibrillator; RVR, rapid ventricular response; $C H F$, congestive heart failure.

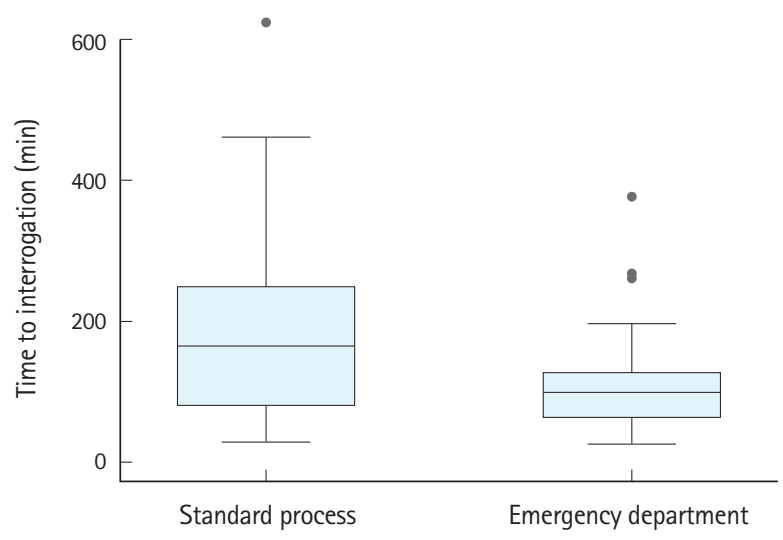

Fig. 2. Time to device interrogation, utilizing standard box plot reporting. The central line represents the median value for each group, the box represents the 25th and 75th percentile distribution respectively. Each whisker then represents the highest (or lowest) value within 1.5 times the intraquartile range, as per the Tukey method. Finally, remaining outliers are documented. For this graph, there was an extreme outlier in the standard process group at 2,485 minutes that is not shown due to resultant distortion $(\mathrm{P}=0.013)$.

to 333$)$ minutes $(P=0.71)$. These results did not vary by time of presentation.

Overall, eleven patients (18\%), 5 ED interrogation and 6 SP patients $(P=0.562)$, required device reprogramming during the index encounter (8 pacemakers and 3 ICDs). No patient in either the ED or SP cohorts suffered an unplanned medical contact or post-discharge adverse event due to the interrogation.

ED interrogations were capable of obtaining all the necessary information for device management which was verified by a company representative at study conclusion.

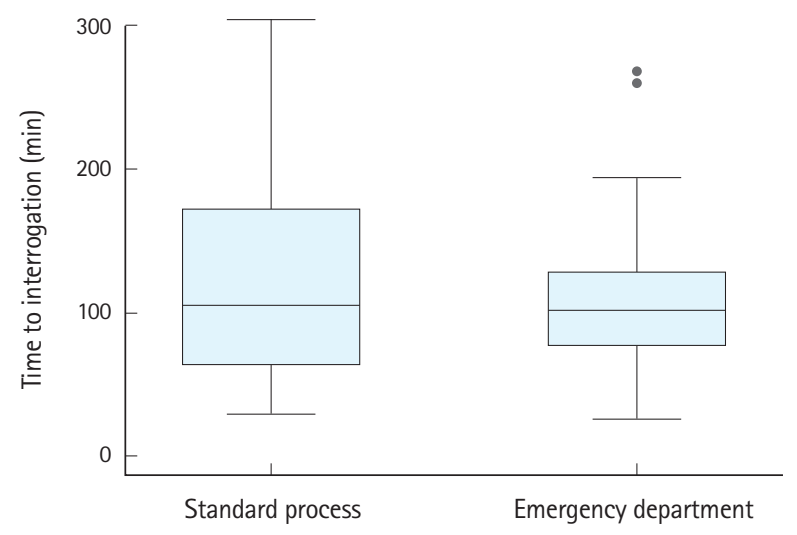

Fig. 3. Time to device interrogation in minutes during business hours. Please see Fig. 2 legend for box plot explanation ( $P=0.62)$.

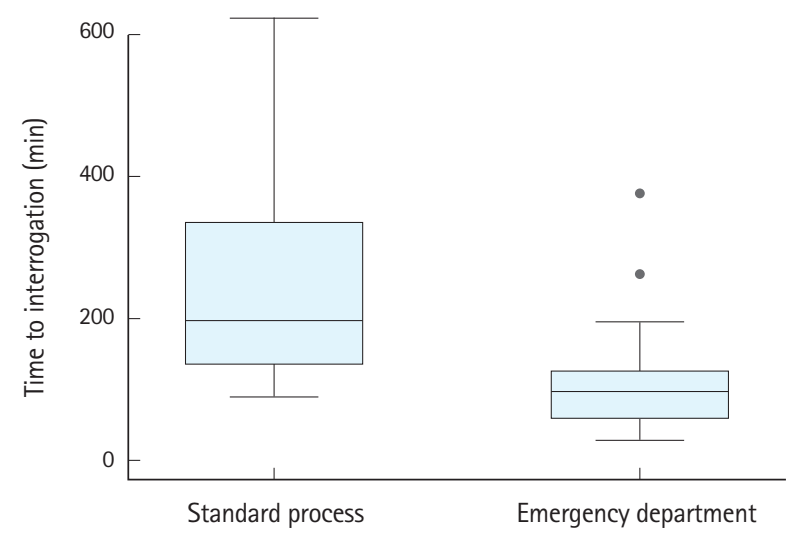

Fig. 4. Time to device interrogation in minutes during off-hours. Please see Fig. 2 legend for box plot explanation ( $P=0.002)$.

\section{DISCUSSION}

We found that time to device interrogation was shorter, both statistically and in a clinically relevant amount, when performed by ED personnel as opposed to having the current SP of having to call external resources to come to the ED. This finding was primarily driven by those patients that presented during off-hours. The interrogations performed by ED personnel typically took less than 10 minutes which is consistent with our previous finding. ${ }^{8}$ Another reason for the ED interrogations being done in less time was because some of the SP group were admitted to the hospital and did not have the interrogation until they left the ED.

We also found that the result of most interrogations was the provision of diagnostic device data, and only required device reprogramming in 18\% of all patients. This suggests that the majority of patients could be managed with data acquisition only, which we have demonstrated can be performed by ED personnel. The capability of doing interrogations in the ED has the potential to decrease transfers from hospitals that currently do not have 
availability to do the interrogation or do not feel comfortable keeping the patient in case a programming adjustment needs to occur. The presence or absence of a cardiac device-related diagnosis could be established quickly with an ED interrogation, potentially avoiding a medically unnecessary transfer.

Although we used only one manufacturer's devices in this study, we utilized a different manufacturer's devices in our previous work. ${ }^{8}$ As both studies showed ED personnel could work with both companies' equipment, we suggest this data can be extrapolated to work for all 3 manufacturers. The only difference between groups was meant to be who gathered the data. Part of the safety evaluation was to measure if the ED arm would obtain all the needed data. In every patient of the ED arm, the ED interrogation was believed to have captured all the necessary information for device management. Although there was no gold standard comparator interrogation in the ED group, we utilized a pragmatic, patient centered standard consisting of follow up with patients' cardiologists for evidence that all information had been gathered and evidence of adverse events.

While improving ED efficiency is generally of benefit, in this small pilot trial, the reduction in time-to-interrogation did not translate into shorter disposition times or an overall decreased ED LOS. Part of this explanation owes to having so few nurses on duty during the study that were capable of doing the consent and operating the programmer. As they often had other responsibilities, delays in getting the consent and interrogations done may have lead to increases in ED LOS. After the identification and consent were in place, the actual interrogation only took about 10 minutes. Also, given that a substantial majority of patients in both study arms were admitted or transferred to a tertiary hospital, this suggests that other operational confounders, i.e., diagnostic, treatment, and administrative processes, are important in determining throughput. Confounding of our ED LOS analysis may have occurred as a result of the ED physician who because of the significant delay that may be associated with non-business hour interrogation requests, would hospitalize the patient for interrogation. While this work around may improve ED flow (and markedly increase costs), it artificially shortens the SP population's ED LOS.

In our previous work, ${ }_{1}^{8}$ we established that ED interrogation provided confirmatory or new diagnostic information in 60\% of patients presenting to the ED, regardless of complaint, with an implanted cardiac device. There are many data elements that can be extracted from implanted cardiac devices that could affect ED management. Of primary concern is the presence or absence of defibrillation in patients presenting with the perception that they have been shocked. Not infrequently, these patients turn out to have experienced a "phantom shock" and did not actually experi- ence defibrillation at all. ${ }^{9}$ Additionally, data on atrial fibrillation burden, degree of physical activity of the patient and the intrathoracic impedance as a measure of pulmonary congestion can help characterize the presence and degree of heart failure in patients whose devices have that capability. ${ }^{10}$

Previously, only medical staff that had extensive training in the utilization of programmers provided interrogation services for pacemakers and ICDs, potentially leading to long delays in care if those personnel were not immediately available to come to the ED. Clinicians are also faced with the issue of cross-platform incompatibility-a cardiac device can only be interrogated by a programmer from the same manufacturer. It is our clinical experience that patients are frequently unclear as to what type of device they have, nor is the information always available in the patient's record, especially when the device was implanted at another facility. While ideally patients are supposed to carry a card identifying the manufacturer and device type, this does not always occur. Delays can be compounded if the wrong manufacturer representative is called in, only to discover that a second representative from a different manufacturer will need to be summoned. Although the major manufacturers maintain registries that can be called for verification, this can be time consuming. All this compounds the issue of ED crowding which is already leading to decreased quality of care, ${ }_{1}^{6}$ increased mortality, ${ }_{1}^{11}$ and emergency medical service diversion. ${ }^{12}$

In the future "read only devices" may prove to decrease time to interrogation and ED LOS, but future studies are needed to confirm or refute this hypothesis. A registry might be a helpful way to collect and accumulate the data needed.

Our study has several limitations. The sample size is small so the adverse event rate may be underestimated. We utilized only one manufacturer and were in one hospital system so results may not be generalizable. Other cofounders may have played roles in time to disposition. The study population was homogenous and may not be representative of the broader population.

In conclusion, ED performed device interrogations decrease the time to data acquisition compared to SPs without increased risk to the patient. This difference is magnified during off-hour and weekend presentations. While interrogations were done sooner, ED LOS was not affected. Further research is required to identify the characteristics of those ED patients who would experience an overall benefit from ED interrogation of cardiac devices.

\section{CONFLICT OF INTEREST}

An unrestricted institutional research grant from Boston Scientific was used to fund the study. Dr. Neuenschwander has consult- 
ing agreements with Boston Scientific and Medtronic and a research grant from St. Jude Medical.

\section{REFERENCES}

1. Greenspon AJ, Patel JD, Lau E, et al. Trends in permanent pacemaker implantation in the United States from 1993 to 2009: increasing complexity of patients and procedures. J Am Coll Cardiol 2012;60:1540-5.

2. Mond HG, Proclemer A. The 11th world survey of cardiac pacing and implantable cardioverter-defibrillators: calendar year 2009: a World Society of Arrhythmia's project. Pacing Clin Electrophysiol 2011;34:1013-27.

3. Moss AJ, Hall WJ, Cannom DS, et al. Improved survival with an implanted defibrillator in patients with coronary disease at high risk for ventricular arrhythmia. Multicenter Automatic Defibrillator Implantation Trial Investigators. N Engl J Med 1996;335:1933-40.

4. Mark DB, Anstrom KJ, Sun JL, et al. Quality of life with defibrillator therapy or amiodarone in heart failure. N Engl J Med 2008;359:999-1008.

5. Bayley MD, Schwartz JS, Shofer FS, et al. The financial burden of emergency department congestion and hospital crowding for chest pain patients awaiting admission. Ann Emerg Med 2005;45:110-7.
6. Pines JM, Hollander JE. Emergency department crowding is associated with poor care for patients with severe pain. Ann Emerg Med 2008;51:1-5.

7. Sprivulis PC, Da Silva JA, Jacobs IG, Frazer AR, Jelinek GA. The association between hospital overcrowding and mortality among patients admitted via Western Australian emergency departments. Med J Aust 2006;184:208-12.

8. Neuenschwander JF, Hiestand BC, Peacock WF, et al. A pilot study of implantable cardiac device interrogation by emergency department personnel. Crit Pathw Cardiol 2014;13:6-8.

9. Starrenburg A, Kraaier K, Pedersen S, Scholten M, Van Der Palen J. Psychological indices as predictors for phantom shocks in implantable cardioverter defibrillator recipients. Pacing Clin Electrophysiol 2014;37:768-73.

10. Hiestand B, Laribi S, Mebazaa A. Implantable cardiac devices and the acute care management of decompensated heart failure. Curr Emerg Hosp Med Rep 2013;1:105-11.

11. Bernstein SL, Aronsky D, Duseja R, et al. The effect of emergency department crowding on clinically oriented outcomes. Acad Emerg Med 2009;16:1-10.

12. American Hospital Association. Taking the pulse: the state of America's hospitals [Internet]. Chicago, IL: American Hospital Association; 2005 [cited 2016 Mar 30]. Available from: http:// www.aha.org/content/00-10/TakingthePulse.pdf. 